BMJ Open

Diabetes

Research

\& Care

\title{
Impaired brain fractalkine-CX3CR1 signaling is implicated in cognitive dysfunction in diet-induced obese mice
}

\author{
Namiko Kawamura (D) , ${ }^{1}$ Goro Katsuura, ${ }^{1}$ Nobuko Yamada-Goto, ${ }^{2,3}$ Ela Novianti, ${ }^{1}$ \\ Akio Inui, ${ }^{4}$ Akihiro Asakawa ${ }^{1}$
}

To cite: Kawamura N, Katsuura G, Yamada-Goto N, et al. Impaired brain fractalkine-CX3CR1 signaling is implicated in cognitive dysfunction in diet-induced obese mice. BMJ Open Diab Res Care 2021;9:e001492. doi:10.1136/ bmjdrc-2020-001492

Received 22 April 2020 Revised 9 December 2020 Accepted 9 January 2021

\section{Check for updates}

\section{(C) Author(s) (or their} employer(s)) 2021. Re-use permitted under CC BY-NC. No commercial re-use. See rights and permissions. Published by BMJ.

${ }^{1}$ Department of Psychosomatic Internal Medicine, Kagoshima University Graduate School of Medical and Dental Sciences, Kagoshima, Japan

${ }^{2}$ Health Center, Keio University, Shinjuku-ku, Tokyo, Japan ${ }^{3}$ Division of Endocrinology, Metabolism and Nephrology, Department of Internal Medicine, Keio University, School of Medicine, Shinjukuku, Tokyo, Japan

${ }^{4}$ Pharmacological Department of Herbal Medicine, Kagoshima University Graduate School of Medical and Dental Sciences, Kagoshima, Japan

Correspondence to Dr Namiko Kawamura; nkawamu@m3.kufm. kagoshima-u.ac.jp

\section{ABSTRACT}

Introduction A diet high in saturated fat is well known to affect neuronal function and contribute to cognitive decline in experimental animals and humans. Fractalkine released from neurons acts on its receptor, CX3C chemokine receptor 1 (CX3CR1), in the microglia to regulate several brain functions. The present study addressed whether fractalkine-CX3CR1 signaling in the brain, especially the hippocampus, contributes to the cognitive deficits observed in diet-induced obese (DI0) mice.

Research design and methods Mice were given $60 \%$ high-fat diet for 16 weeks. The expression of fractalkine and CX3CR1 in the hippocampus, amygdala and prefrontal cortex of DIO mice was analyzed. Cognitive ability in the Y-maze test and hippocampal glutamate receptors and synaptic markers were observed in DI0 and CX3CR1 antagonist-treated mice. Regulation of fractalkine and CX3CR1 expression in the hippocampus was examined following administration of a selective insulin-like growth factor-1 (IGF-1) receptor inhibitor and a tyrosine receptor kinase $B($ TrkB) antagonist in normal mice.

Results DI0 mice exhibited significant cognitive deficits in the Y-maze test and decrease in fractalkine and CX3CR1 in the hippocampus and amygdala compared with mice fed a control diet (CD mice). Administration of the CX3CR1 antagonist 18a in normal mice induced significant cognitive deficits in the Y-maze test. DIO mice and CX3CR1 antagonist-treated mice exhibited significant decreases in protein levels of NMDA (N-methyl-D-aspartate) receptor subunit (NR2A), AMPA ( $\alpha$-amino-5-methyl-3-hydroxy4-isoxazole propionate) receptor subunit (GluR1) and postsynaptic density protein 95 in the hippocampus compared with their respective controls. Furthermore, plasma IGF-1 and hippocampal brain-derived neurotrophic factor were significantly decreased in DIO mice compared with CD mice. Administration of a selective IGF-1 receptor inhibitor and a TrkB antagonist in normal mice significantly decreased fractalkine and CX3CR1 in the hippocampus. Conclusions These findings indicate that the cognitive decline observed in DIO mice is due, in part, to reduced fractalkine-CX3CR1 signaling in the corticolimbic system.

\section{INTRODUCTION}

Obesity is associated with a higher risk of lifestyle-related cardiovascular and metabolic disorders, such as hypertension, diabetes, and hyperlipidemia.

\section{Significance of this study}

What is already known about this subject?

- Epidemiological studies indicate that the incidence of cognitive impairment is higher in obese subjects than in those with normal body weight.

- Diet-induced obese (DI0) animals exhibit cognitive deficits in a variety of behavioral tests.

- Fractalkine released from neurons acts on its receptor, CX3C chemokine receptor 1 (CX3CR1), in the microglia to regulate several brain functions.

- CX3CR1 deficiency induces impairment of hippocampal cognitive function and synaptic plasticity.

What are the new findings?

- DIO mice exhibited significant decrease in fractalkine and CX3CR1 in the hippocampus and amygdala, as well as significant cognitive deficits in the Y-maze test, compared with mice fed a control diet (CD mice).

- Administration of the CX3CR1 antagonist 18a in normal mice induced significant cognitive deficits in the Y-maze test.

- DIO mice and CX3CR1 antagonist-treated mice exhibited significant decreases in protein levels of NMDA (N-methyl-D-aspartate receptor) subunit (NR2A), AMPA ( $\alpha$-amino-5-methyl-3-hydroxy-4isoxazole propionate) receptor subunit (GluR1) and postsynaptic density protein 95 in the hippocampus compared with their respective controls.

- Plasma insulin-like growth factor-1 (IGF-1) and hippocampal brain-derived neurotrophic factor were significantly decreased in DIO mice compared with CD mice.

- Administration of a selective IGF-1 receptor inhibitor and a tyrosine receptor kinase $B(T r k B)$ antagonist in normal mice significantly decreased fractalkine and CX3CR1 in the hippocampus.

\section{How might these results change the focus of} research or clinical practice?

- These findings provide compelling evidence that neuron-microglia interactions, especially those underpinned by fractalkine-CX3CR1 signaling, play crucial roles in brain dysfunction associated with obesity.

Epidemiological studies indicate that the incidence of cognitive impairment is higher in obese subjects than in those with 
normal body weight. ${ }^{1}{ }^{2}$ Diet-induced obese (DIO) animals exhibit impaired cognition in a variety of behavioral tests, such as the Morris water maze and a spontaneous alternation paradigm in T-maze and Y-maze tests. ${ }^{3-6}$ Consistent with these findings, our previous study demonstrated that DIO mice fed a high-fat diet (HFD) exhibit significant impairments in hippocampus-dependent and amygdala-dependent fear-conditioning responses and have low levels of hippocampal brain-derived neurotrophic factor (BDNF), which plays a critical role in synaptic plasticity, long-term potentiation (LTP) and learning and memory processes. ${ }^{7}$ Furthermore, electrophysiological studies indicate that LTP in the hippocampal CA1 region, which is closely related to memory formation and is predominately regulated by the glutamatergic system, especially N-methyl-D-aspartate (NMDA) and $\alpha$-amino-5-methyl-3-hydroxy-4-isoxazole propionate (AMPA) receptors, ${ }^{8}$ is markedly impaired in DIO animals compared with lean animals. ${ }^{9} 10$ The cellular and molecular events of the synaptic plasticity involved in learning and memory processes are modulated by neurotrophic factors including BDNF and insulin-like growth factor-1 (IGF-1), ${ }^{11-13}$ which regulate the presynaptic and postsynaptic machinery associated with synaptic plasticity. ${ }^{6914}$

Communication between neurons and microglia is crucial for the optimal regulation of central nervous system (CNS) activities. In this regard, fractalkine secreted from neurons was recently reported to bind to CX3C chemokine receptor 1 (CX3CR1), which is expressed mainly in the microglia, ${ }^{15-17}$ and to regulate several functions of the CNS. ${ }^{18}$ In the mouse brain, fractalkine messenger RNA (mRNA) levels are high in the cortex, hippocampus, and striatum; intermediate in the olfactory bulb, thalamus, hypothalamus and brainstem; and low in the cerebellum. ${ }^{15} 16$ Therefore, fractalkine-CX3CR1 signaling is postulated to represent a primary neuron-glia inter-regulatory system that is important for brain function. ${ }^{18}$ Recent evidence indicates that fractalkine-CX3CR1 signaling plays an important role in regulating LTP formation in the hippocampus and behavioral learning and memory processes. ${ }^{19} 20$

To provide a new insight into the understanding of cognitive decline that occurs in obesity, we assessed the possible contribution of impaired fractalkineCX3CR1 signaling in the corticolimbic system, which is an important system for cognitive processing, to the cognitive deficits observed in DIO mice. In addition, to investigate the underlying mechanisms, we examined the expression of glutamate receptor subunits and synaptic components specific to synaptic plasticity. Our findings revealed that decreased fractalkineCX3CR1 signaling in the corticolimbic system contributes to the impaired cognition observed in DIO mice in association with decreased expression of glutamate receptor subunits and synaptic components.

\section{MATERIALS AND METHODS}

\section{Animals}

Male C57BL/6J mice (6 weeks old) were obtained from CLEA Japan (Tokyo, Japan) and housed in plastic cages under a 12:12-hour light:dark cycle (lights turned on at 07:00) at room temperature $\left(23^{\circ} \mathrm{C} \pm 1^{\circ} \mathrm{C}\right)$. The animals had ad libitum access to water and food (CE-2; CLEA Japan). Every effort was made to optimize the comfort of the animals and to minimize their use.

\section{DIO mice}

Normal mice (6 weeks old) were randomly divided into two groups. The first group was given CE-2 as a control diet (CD), with fat accounting for $12.6 \%$ of the total calories $(343.1 \mathrm{kcal} / 100 \mathrm{~g})$. The second group was given HFD (No. D12492; Research Diets, New Brunswick, New Jersey), with fat accounting for $60 \%$ of the total calories, predominantly in the form of lard $(524 \mathrm{kcal} / 100 \mathrm{~g})$. After feeding on the diets for 16 weeks, we evaluated them in the Y-maze test and measured their body weight before the mice were sacrificed to collect blood samples, fat tissues and brain regions under isoflurane anesthesia.

\section{Analysis of metabolic parameters and sampling of the brain regions}

Blood samples were collected from the retro-orbital vein under isoflurane anesthesia and immediately transferred to tubes containing EDTA ( $10 \mu \mathrm{L}$ of $0.2 \mathrm{M}$ EDTA/tube) and aprotinin $(0.1 \mathrm{mg} /$ tube; Merck KGaA, Darmstadt, Germany). The blood samples were centrifuged $3000 \times g$ for $5 \mathrm{~min}$ at $4^{\circ} \mathrm{C}$, and plasma was separated and stored at $-80^{\circ} \mathrm{C}$ until assayed. After blood collection, the mice were killed by decapitation. The brain was rapidly removed from the skull and placed on an ice-cooled paraffin plate for dissection of the hippocampus, amygdala and prefrontal cortex (PFC) by referring to the mouse brain atlas as previously described. ${ }^{21}$ The brain regions were immediately frozen in liquid nitrogen and stored at $-80^{\circ} \mathrm{C}$ until analyzed. The epididymal and mesenteric fat tissues were collected and weighed. Glucose (Glucose C2; FUJIFILM Wako Pure Chemical Corporation, Osaka, Japan), insulin (Morinaga Ultra Sensitive Mouse/Rat Insulin ELISA Kit; Morinaga Institute of Biological Science, Yokohama, Japan), leptin (Mouse Leptin ELISA; Linco Research, St Charles, Missouri), IGF-1 (Mouse/ Rat IGF-1 Quantikine ELISA Kit; R\&D Systems, Minneapolis, Minnesota) and BDNF (Mouse/Rat BDNF Emax ImmunoAssay System; Promega Corporation, Madison, Wisconsin) were measured using commercially available kits.

\section{Y-maze test}

Cognitive ability was assessed using the Y-maze test, which is generally used to evaluate learning and memory ability in experimental animals, such as mouse models of obesity, Alzheimer's disease, and cerebral ischemia. ${ }^{622}{ }^{23}$ This task is based on the observation that if a mouse remembers the arm that has been explored most recently, a mouse will 
next enter an arm of the maze that has not been visited yet or the most remotely visited arm. ${ }^{24}$ The Y-maze apparatus (Muromachi, Tokyo, Japan) comprises three gray plastic arms (each one $41.5 \mathrm{~cm}$ long, $10 \mathrm{~cm}$ high, and $4 \mathrm{~cm}$ wide) that emanate from the center of the maze, are separated by $120^{\circ}$, and labeled as A, B, and C. Briefly, each mouse was placed at the end of one arm and allowed to move freely for 8 min session without any stimulation. ${ }^{25}$ The sequence of arm entries was recorded manually, with an entry defined as all four limbs of the mouse within an arm. After completing the test trial for each mouse, the maze was cleaned with $70 \%$ ethyl alcohol to remove the mouse odor. The outcomes included the percentage of spontaneous alternations and the number of arm entries. The recorded spontaneous alternation behavior was used to assess hippocampal-dependent spatial memory. The percentage of spontaneous alternations was calculated as the ratio of arm entries that differed from the previous two arm entries (actual alternations) to the total possible alternations (defined as the total entries minus one) and multiplied by 100 .

\section{Reverse transcription-polymerase chain reaction (RT-PCR)}

The mRNA levels were measured by quantitative realtime RT-PCR. ${ }^{26}$ All gene-specific mRNA expression values were normalized against the internal housekeeping gene, glyceraldehyde-3-phosphate dehydrogenase (GAPDH). Primers for GAPDH were as follows: (sense TGCACCACCAACTGCTTAGC, antisense GGATGCAG GGATGATGTTCTG), for fractalkine (sense ACGAAATG CGAAATCATGTGC, antisense CTGTGTCGTCTCCAGGACAA), for CX3CR1 (sense CGTGAGACTGGGTGAGTGAC, antisense AAGGAGGTGGACATGGTGAG), for NR1 (sense AAGCCCAACGCCATACAGAT, antisense AGGCGGGTGACTAACTAGGA), for NR2A (sense TCACTGAGGAAGGCTATC, antisense CCCACTTGCCCACCTTTT), for NR2B (sense AGAGTCGACGAG CTGAAGATGAAGCCCAGC, antisense CGGGGAAC TACTGAGAGATGATGGAAGTCA), for GluR1 (sense GGAGTGGAAGTCCCTAGCACACA, antisense CCTG GGAGTGGCTGCATAAGA), for GluR2 (sense ATGG AACATTAGACTCTGGCTCCAC, antisense CTGCCGTA GTCCTCACAAACACA), for synaptophysin (sense CCACCTCCTTCTCCAATCAG, antisense CAGCAAAGACAGGGTCTCCT), and for postsynaptic density protein 95 (PSD-95) (sense CGAGGAGCCGTGGCAGCC, antisense CATGGCTGTGGGGTAGTCAGTGCC).

\section{Western blotting analysis}

The hippocampus was homogenized in $500 \mu \mathrm{L}$ of cold RIPA buffer $(150 \mathrm{mM} \mathrm{NaCl}, 50 \mathrm{mM}$ Tris, $5 \mathrm{mM}$ EDTA, $50 \mathrm{mM}$ $\mathrm{NaF}, 10 \mathrm{mM}$ sodium pyrophosphate, $1 \mathrm{mM}$ sodium orthovanadate, $1 \% \mathrm{NP}-40,0.5 \%$ deoxycholate, $0.1 \%$ sodium dodecyl sulfate (SDS) (pH 7.5), supplemented with $1 \mathrm{mM}$ leupeptin, $1 \mu \mathrm{g} / \mathrm{mL}$ aprotinin and $1 \mathrm{mM}$ phenylmethylsulfonyl fluoride) as described previously. ${ }^{27}$ The membrane was incubated overnight in Tris-buffered saline with $0.1 \%$ Tween 20, supplemented with 5\% skim milk powder at $4^{\circ} \mathrm{C}$ with the primary antibody as follows: rabbit polyclonal
anti-CX3CL1 (fractalkine) antibody (1:1000, ab25088; abcam, Cambridge, UK) which recognizes the soluble form of fractalkine, mouse monoclonal anti-CX3CR1 antibody (1:250, ab184678; abcam), rabbit monoclonal anti-NMDA receptor 1 (GluN1) (D65B7) antibody (1:1000, \#5704; Cell Signaling Technology, Danvers, Massachusetts), rabbit polyclonal anti-NMDA receptor 2A (GluN2A) antibody (1:1000, \#4205; Cell Signaling Technology), rabbit monoclonal antiNMDA receptor 2B (GluN2B) (D8E10) antibody (1:1000, \#14544; Cell Signaling Technology), rabbit polyclonal antiglutamate receptor 1 (AMPA subtype) antibody (1:1000, ab31232; abcam), rabbit monoclonal anti-AMPA receptor 2 (GluA2) (E1L8U) antibody (1:1000, \#13607; Cell Signaling Technology), rabbit monoclonal anti-synaptophysin (D8F6H) XP antibody (1:1000, \#36406; Cell Signaling Technology), rabbit monoclonal anti-PSD-95 (D27E11) XP antibody (1;1000, \#3450; Cell Signaling Technology) and mouse monoclonal anti-GAPDH (1:2000, sc-32233; Santa Cruz Biotechnology, Dallas, Texas) antibody. Next, the membrane was incubated for 1 hour at room temperature with either anti-rabbit IgG antibody (NA934; GE HealthCare UK, Buckinghamshire, UK) conjugated to horseradish peroxidase or anti-mouse IgG antibody (NA931; GE HealthCare UK) conjugated to horseradish peroxidase at a 1:1000 dilution, followed by detection using the ECL (GE HealthCare UK). The LAS-1000 image analyzer (V.4.0; FUJIFILM Corporation, Tokyo, Japan) was used for detection and quantification. To ensure equivalent amounts of loaded proteins and quantify targeted protein expression, the ratio of the targeted protein level to the GAPDH level was determined.

\section{Intracerebroventricular administration of the CX3CR1 antagonist 18a}

A highly selective antagonist for CX3CR1, 18a (Axon Medchem, Groningen, The Netherlands), with a Ki value of $3.9 \mathrm{nM}$, was dissolved in dimethyl sulfoxide (DMSO), and diluted in saline to a final concentration of $0.1 \%$ DMSO. ${ }^{28}$ The CX3CR1 antagonist $18 \mathrm{a}$ ( $\left.50 \mathrm{ng} / \mathrm{mouse}\right)$ was intracerebroventricularly administrated at 1 hour before the Y-maze test according to our previous report. ${ }^{27}$ Mice in the control group were given intracerebroventricular injections of an equal volume of vehicle $(0.1 \%$ DMSO in saline, $2 \mu \mathrm{L} /$ mouse). In another experiment, mice were sacrificed at 1 hour after the administration to collect brain regions under isoflurane anesthesia.

\section{Chronic intraperitoneal administration of picropodophyllin and ANA-12}

The selective IGF-1 receptor inhibitor picropodophyllin (PPP, $20 \mathrm{mg} / \mathrm{kg}$; Santa Cruz Biotechnology) ${ }^{29}$ and the tyrosine receptor kinase B (TrkB) selective and noncompetitive antagonist ANA-12 $(0.5 \mathrm{mg} / \mathrm{kg} ;$ Merck $\mathrm{KGaA})^{30}$ were dissolved in DMSO and diluted with saline at a final concentration of 50\% DMSO. PPP and ANA-12 were intraperitoneally injected at 10:00 once a day for 7 days. Mice in the control group were given an intraperitoneal injection of an equal volume of vehicle (50\% DMSO in saline, $10 \mathrm{~mL} / \mathrm{kg}$ body weight). Mice were sacrificed 
(A) Body weight

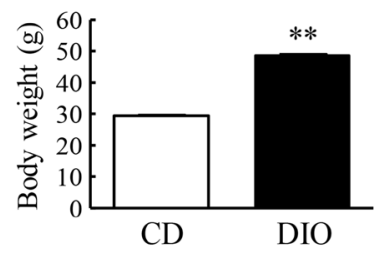

(C) Mesenteric fat

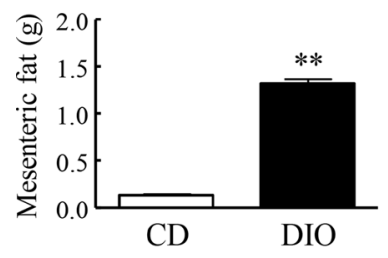

(E) Plasma insulin

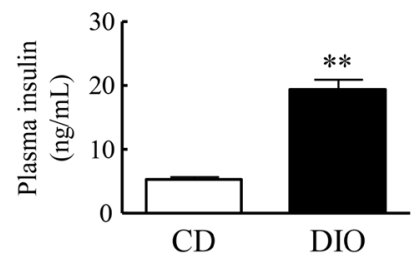

(G) Plasma IGF-1

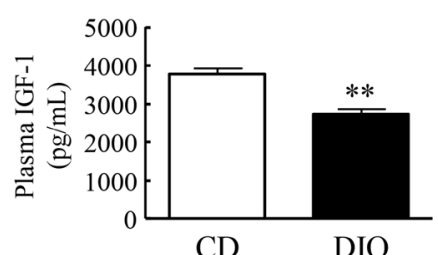

CD DIO
(B) Epididymal fat

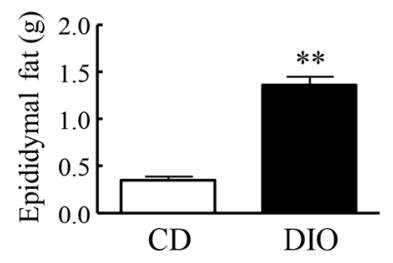

(D) Plasma glucose

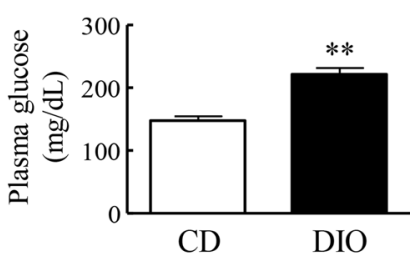

(F) Plasma leptin

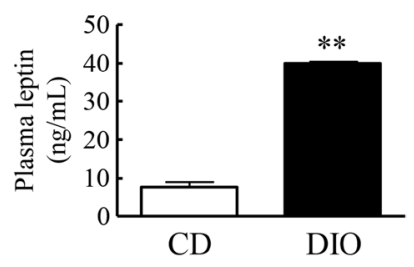

(H) Plasma BDNF

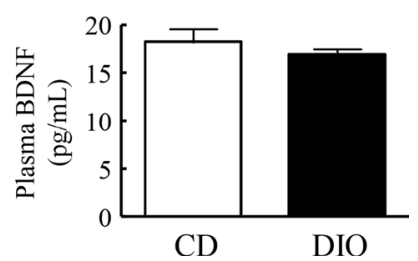

Figure 1 Changes in body weight, fat mass and plasma levels of glucose, insulin, leptin, IGF-1 and BDNF in DIO mice. (A) Body weight, (B) epididymal fat, (C) mesenteric fat, (D) plasma glucose levels, (E) plasma insulin levels, (F) plasma leptin levels, (G) plasma IGF-1 levels, and (H) plasma BDNF levels. Results are expressed as mean \pm SE for 5-17 mice. ${ }^{* *} P<0.01$ versus $C D$. BDNF, brain-derived neurotrophic factor; CD, control diet; DIO, diet-induced obese; IGF-1, insulin-like growth factor-1.

at 1 hour after the last administration to collect brain regions under isoflurane anesthesia.

\section{Data analysis}

Data are expressed as mean \pm SEM. Statistical analysis of the data was performed by analysis of variance followed by the Tukey-Kramer test. Statistical significance was defined as $\mathrm{p}<0.05$.

\section{RESULTS}

Changes in body weight, fat mass, and plasma levels of glucose, insulin, leptin, IGF-1 and BDNF in DI0 mice

DIO mice had markedly greater body weight and epididymal and mesenteric fat mass than normal mice fed CD (CD mice) (figure 1A-C). Plasma levels of glucose, insulin and leptin were significantly increased in DIO mice compared with CD mice (figure 1D-F). Plasma IGF-1 levels in DIO

mice were significantly decreased to $73 \%$ of that in CD mice, and plasma BDNF levels did not differ significantly between DIO mice and CD mice (figure $1 \mathrm{G}, \mathrm{H}$ ).

\section{Changes in expression of fractalkine, CX3CR1, IGF-1 and} BDNF in the brain of DI0 mice

To explore the possible involvement of fractalkineCX3CR1 signaling in the cognitive deficits observed in DIO mice, we examined changes in fractalkine and CX3CR1 expression in the hippocampus, amygdala and $\mathrm{PFC}$ of DIO mice compared with CD mice. Moreover, we examined the hippocampal IGF-1 and BDNF levels, which are well known to play a pivotal role in learning and memory. ${ }^{11-13}$ The mRNA expression of fractalkine was significantly decreased in the hippocampus of DIO mice to $79 \%$ of that in $\mathrm{CD}$ mice (figure $2 \mathrm{~A}$ ). The protein levels of soluble form of fractalkine were significantly decreased in the hippocampus of DIO mice to $85 \%$ of those in CD mice (figure 2B). The mRNA expression and protein levels of CX3CR1 were significantly decreased in the hippocampus of DIO mice to $75 \%$ and $56 \%$, respectively, of those in $\mathrm{CD}$ mice (figure 2C,D). In addition, protein levels of BDNF, but not IGF-1, in the hippocampus of DIO mice were significantly decreased to $78 \%$ of that in $\mathrm{CD}$ mice (figure 2E,F). The mRNA expression of fractalkine and CX3CR1 was significantly decreased in the amygdala of DIO mice to $86 \%$ and $79 \%$, respectively, of that in $\mathrm{CD}$ mice (figure $2 \mathrm{G}$ ). On the other hand, the mRNA expression of fractalkine and CX3CR1 in the PFC was not changed in DIO mice (figure $2 \mathrm{H}$ ).

\section{Cognitive ability in the Y-maze test and changes in mRNA} expression and protein levels of NMDA and AMPA receptor subunits, synaptophysin and PSD-95 in the hippocampus in DIO mice

Spontaneous alternation in the Y-maze test was significantly reduced in DIO mice to $76 \%$ of that in CD mice (figure 3A). The number of arm entries was not significantly different between DIO mice and CD mice (figure 3B).

To elucidate the possible mechanisms underlying cognitive impairment in DIO mice, we examined the hippocampal expression of NMDA receptor subunits, AMPA receptor subunits, synaptophysin and PSD-95. The mRNA expression of NR2A, but not NR1 and NR2B, of NMDA receptor subunits in the hippocampus of DIO mice was significantly decreased to $68 \%$ of the levels in CD mice (figure 3C,E,G). The protein levels of NR1 and NR2A in the hippocampus of DIO mice were significantly decreased to $62 \%$ and $58 \%$, respectively, of the levels in $\mathrm{CD}$ mice (figure 3D,F). The protein levels of NR2B tended to be decreased to $85 \%$ of the levels in CD mice (figure $3 \mathrm{H}$ ). The mRNA expression and protein levels of GluR1, but not GluR2, of AMPA receptor subunits in the hippocampus of DIO mice were significantly decreased to $81 \%$ and $74 \%$, respectively, of the levels in CD mice (figure 3I-L). The protein levels, but not mRNA expression, of synaptophysin and PSD-95 in the hippocampus 
(A) Fractalkine mRNA

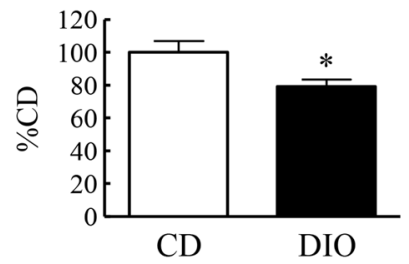

(C) CX3CR1 mRNA

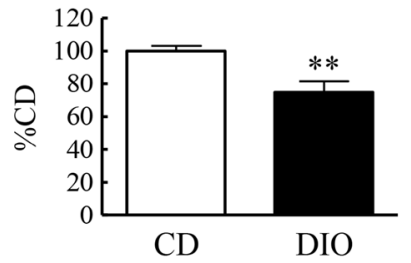

(E) IGF-1 protein

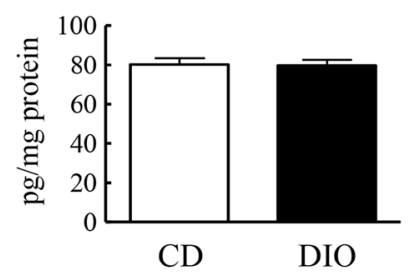

(G) Amygdala

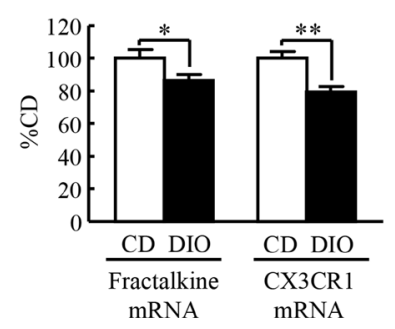

(B) Fractalkine protein

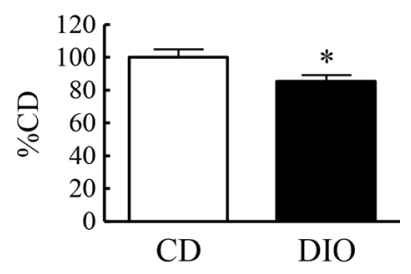

(D) CX3CR1 protein

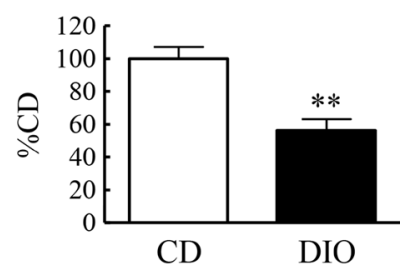

(F) BDNF protein

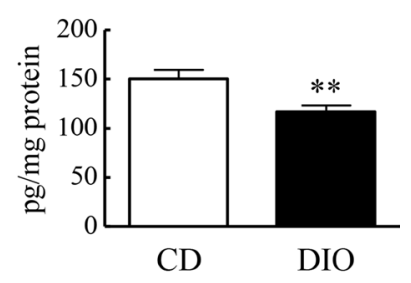

(H) PFC

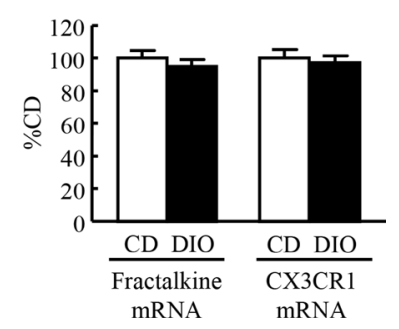

Figure 2 Changes in expression of fractalkine, CX3CR1, IGF-1 and BDNF in the brain of DIO mice. (A) Fractalkine mRNA expression, (B) fractalkine protein levels, (C) CX3CR1 mRNA expression, (D) CX3CR1 protein levels, (E) IGF-1 protein levels, and (F) BDNF protein levels in the hippocampus. (G) Fractalkine and CX3CR1 mRNA expression in the amygdala. $(\mathrm{H})$ Fractalkine and CX3CR1 mRNA expression in the PFC. Results are expressed as mean \pm SE for $5-16$ mice. ${ }^{*} P<0.05$, ${ }^{* \star} P<0.01$ versus $C D$. BDNF, brain-derived neurotrophic factor; CD, control diet; CX3CR1, CX3C chemokine receptor 1; DIO, diet-induced obese; IGF1, insulin-like growth factor-1; mRNA, messenger RNA; PFC, prefrontal cortex.

of DIO mice were markedly decreased to $68 \%$ and $61 \%$, respectively, of the levels in $\mathrm{CD}$ mice (figure $3 \mathrm{M}-\mathrm{P}$ ).

\section{Effects of intracerebroventricular administration of the}

CX3CR1 antagonist 18a on cognitive ability in the Y-maze test and changes in mRNA expression and protein levels of NMDA and AMPA receptor subunits, synaptophysin and PSD-95 in the hippocampus in normal mice

To examine the possible contribution of decreased fractalkine-CX3CR1 signaling to the cognitive impairment observed in DIO mice, we examined the effect of

the CX3CR1 antagonist 18a on learning and memory in the Y-maze test in normal mice. Spontaneous alternation in the Y-maze test was significantly reduced in mice with intracerebroventricular administration of $18 \mathrm{a}$ to $75 \%$ of that in vehicle-treated mice (figure $4 \mathrm{~A}$ ). The number of arm entries was not changed in either of the two groups (figure 4B).

To further evaluate the involvement of fractalkineCX3CR1 signaling in cognitive deficits in DIO mice, the changes in the same factors observed in the hippocampus of DIO mice, as shown in figure 3, were examined in the hippocampus of mice treated with the CX3CR1 antagonist 18a. The mRNA expression of the NR1, but not NR2A and NR2B, in the hippocampus of the antagonist-treated mice was significantly decreased to $67 \%$ of that in vehicle-treated mice (figure 4C,E,G). The protein levels of NR2A, but not NR1 and NR2B, in the hippocampus of the antagonist-treated mice were significantly decreased to $53 \%$ of the levels in vehicle-treated mice (figure 4D,F,H). The mRNA expression and protein levels of the GluR1, but not GluR2, in the hippocampus of the antagonist-treated mice were significantly decreased to $70 \%$ and $62 \%$, respectively, of the levels in vehicletreated mice (figure 4I-L). The mRNA expression and protein levels of synaptophysin in the hippocampus were not changed by administration of $18 \mathrm{a}$ (figure $4 \mathrm{M}, \mathrm{N}$ ). The mRNA expression and protein levels of PSD-95 in the hippocampus of the antagonist-treated mice were significantly decreased to $77 \%$ and $66 \%$, respectively, of the levels in vehicle-treated mice (figure 4O,P).

\section{Regulatory effects of IGF-1 and BDNF on mRNA expression of} fractalkine and CX3CR1 in the hippocampus of normal mice Plasma IGF-1 levels and hippocampal BDNF levels were significantly lower in DIO mice compared with CD mice (figures 1G and 2F). We then examined whether IGF-1 and $\mathrm{BDNF}$ regulate the mRNA expression of fractalkine and CX3CR1 in the hippocampus of normal mice. Chronic intraperitoneal administration of the selective IGF-1 receptor inhibitor PPP induced a significant decrease in the mRNA expression of fractalkine and CX3CR1 in the hippocampus to $79 \%$ and $77 \%$, respectively, of those in vehicle-treated mice (figure 5A,B). Chronic intraperitoneal administration of the TrkB selective and noncompetitive antagonist ANA-12 also induced significant decrease in the mRNA expression of both fractalkine and CX3CR1 in the hippocampus to $83 \%$ and $75 \%$, respectively, of those in vehicle-treated mice (figure 5C,D).

\section{DISCUSSION}

In the present study, DIO mice exhibited significant impairment in learning and memory in the Y-maze test compared with control mice, and a significant reduction in both fractalkine and CX3CR1 levels in the hippocampus and amygdala. Furthermore, intracerebroventricular administration of the CX3CR1 antagonist 18a in normal mice induced significant cognitive deficits in 
(A) Spontaneous alternation

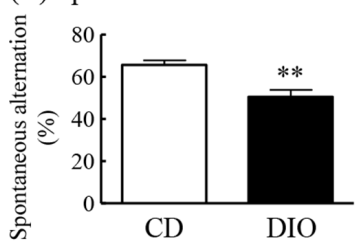

(E) NR2A mRNA

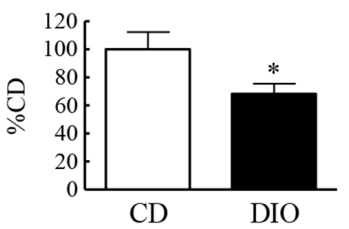

(I) GluR1 mRNA

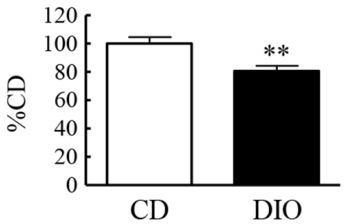

(M) Synaptophysin mRNA

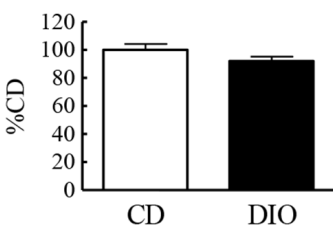

(B) Number of arm entries

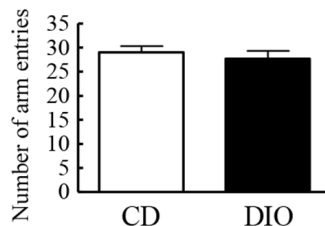

(F) NR2A protein

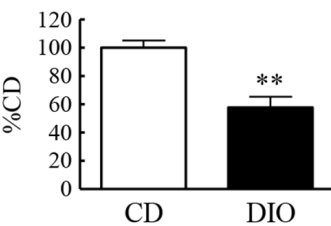

(J) GluR1 protein

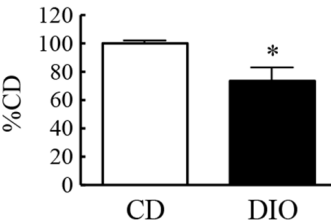

(N) Synaptophysin protein

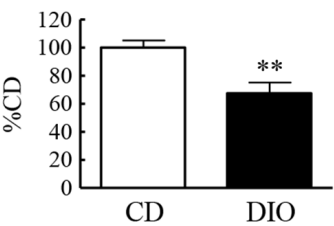

(C) NR1 mRNA

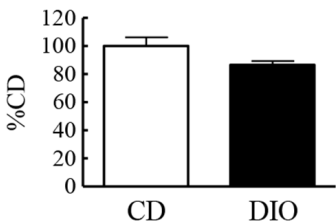

(G) NR2B mRNA

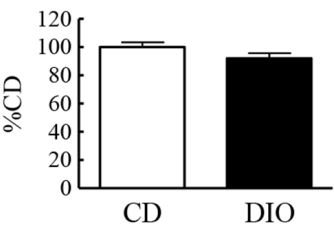

(K) GluR2 mRNA

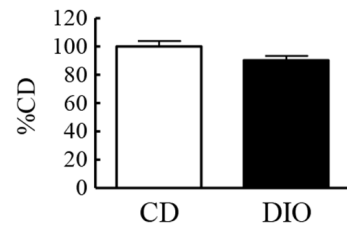

(O) PSD-95 mRNA

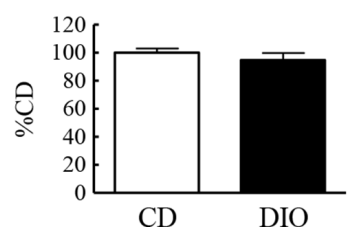

(D) NR1 protein

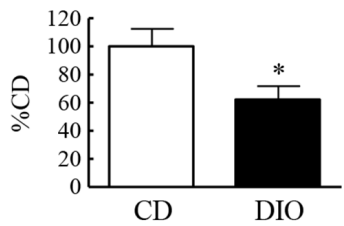

(H) NR2B protein

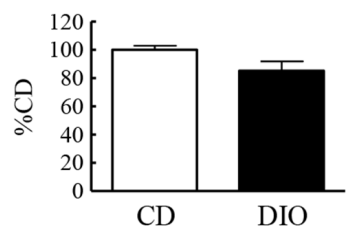

(L) GluR2 protein

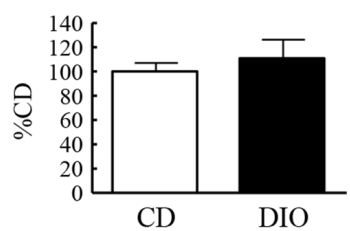

(P) PSD-95 protein

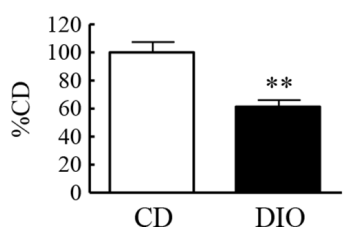

Figure 3 Cognitive ability in the Y-maze test and changes in mRNA expression and protein levels of NMDA and AMPA receptor subunits, synaptophysin and PSD-95 in the hippocampus in DIO mice. Y-maze test: (A) spontaneous alternation (\%) and (B) number of arm entries. NR1: (C) mRNA expression and (D) protein levels. NR2A: (E) mRNA expression and (F) protein levels. NR2B: (G) mRNA expression and (H) protein levels. GluR1: (I) mRNA expression and (J) protein levels. GluR2: (K) mRNA expression and (L) protein levels. Synaptophysin: (M) mRNA expression and (N) protein levels. PSD-95: (O) mRNA expression and $(P)$ protein levels. Results are expressed as mean $\pm S E$ for $5-18$ mice. ${ }^{*} P<0.05,{ }^{\star \star} P<0.01$ versus CD. AMPA, $\alpha$-amino-5methyl-3-hydroxy-4-isoxazole propionate; CD, control diet; DIO, diet-induced obese; mRNA, messenger RNA; NMDA, Nmethyl-D-aspartate; PSD-95, postsynaptic density protein 95.

the Y-maze test. Regarding the mechanism underlying the cognitive deficits in DIO mice, DIO mice exhibited a significant decrease in NR1 and NR2A of NMDA receptor subunits, GluR1 of AMPA receptor subunits, synaptophysin, and PSD-95 in the hippocampus. Moreover, CX3CR1 antagonist-treated mice exhibited a significant decrease in NR2A, GluR1 and PSD-95 in the hippocampus. Furthermore, plasma IGF-1 and hippocampal BDNF were significantly decreased in DIO mice compared with $\mathrm{CD}$ mice. Administration of the IGF-1 receptor inhibitor PPP and the TrkB antagonist ANA-12 significantly decreased mRNA expression of fractalkine and CX3CR1 in the hippocampus of normal mice. These findings suggest that the cognitive decline observed in DIO mice is due, at least in part, to reduced fractalkineCX3CR1 signaling in the corticolimbic system.

A diet high in saturated fat is well known to affect neuronal function and contribute to cognitive decline in experimental animals and humans. ${ }^{10} 31-33$ Obese animals fed over the long term with a diet high in saturated fat exhibit impaired acquisition and retention of spatial memory in the water maze test, and low levels of hippocampal BDNF to the extent that cognitive performance is compromised. ${ }^{34914}$ Our previous study demonstrated that DIO mice fed HFD exhibit significant attenuation of hippocampus-dependent and amygdaladependent fear-conditioning responses and low levels of hippocampal BDNF. ${ }^{7}$ The amygdala regulates hippocampal LTP, spatial memory and dentate gyrus field potentials. ${ }^{3435}$ Moreover, the PFC influences the responsiveness of the central amygdala output neurons. ${ }^{36}$ These findings indicate that hippocampus, amygdala and PFC play an important role in learning and memory processes. Therefore, we examined changes in the expression of fractalkine and CX3CR1 in the hippocampus, amygdala and PFC of DIO mice. The present study provides the first evidence that DIO mice have significant decrease in fractalkine and CX3CR1 levels in the hippocampus and amygdala, and that the administration of a CX3CR1 antagonist significantly impairs learning and memory in the Y-maze test in normal mice, consistent with cognitive deficits in CX3CR1-deficient mice. ${ }^{20}$ Electrophysiological studies demonstrated that LTP in the hippocampal CA1 region is markedly impaired in DIO rats compared with 
(A) Spontaneous alternation

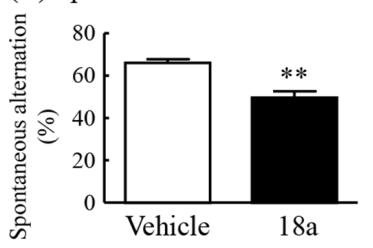

(E) NR2A mRNA

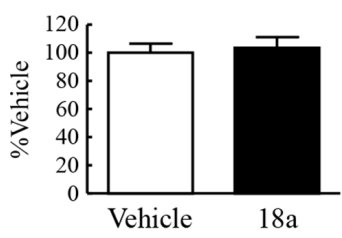

(I) GluR1 mRNA

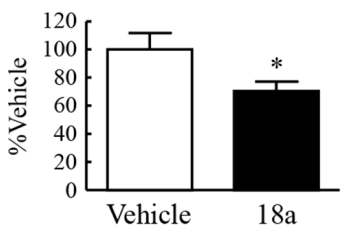

(M) Synaptophysin mRNA

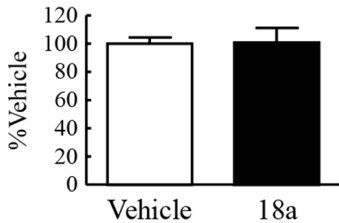

(B) Number of arm entries

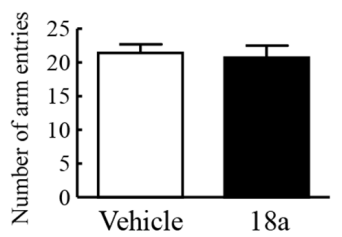

(F) NR2A protein

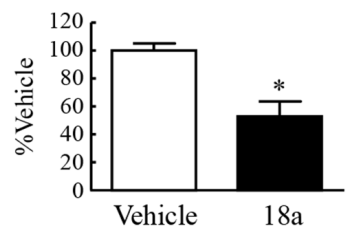

(J) GluR1 protein

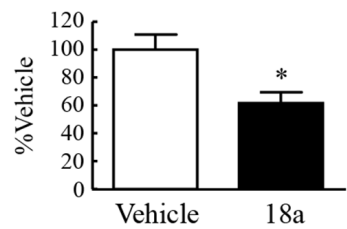

(N) Synaptophysin protein

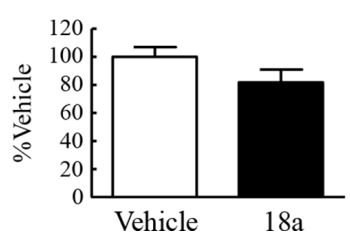

(C) NR1 mRNA

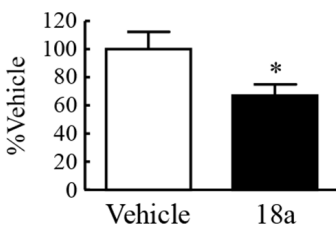

(G) NR2B mRNA

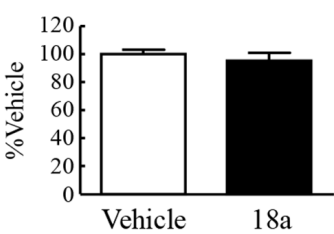

(K) GluR2 mRNA

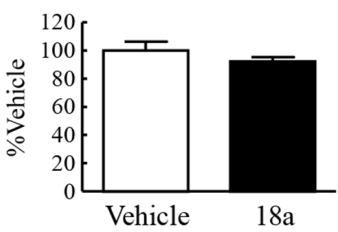

(O) PSD-95 mRNA

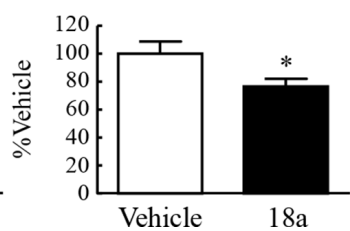

(D) NR1 protein

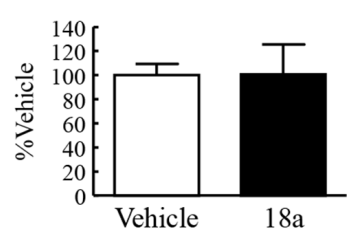

(H) NR2B protein

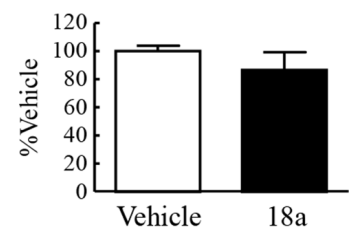

(L) GluR2 protein

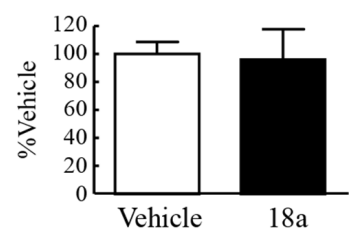

(P) PSD-95 protein

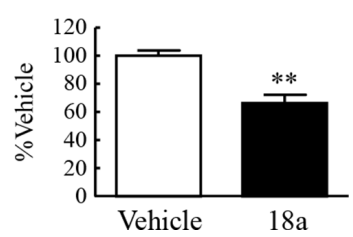

Figure 4 Effects of intracerebroventricular administration of the CX3CR1 antagonist 18A on cognitive ability in the Y-maze test and changes in mRNA expression and protein levels of NMDA and AMPA receptor subunits, synaptophysin and PSD95 in the hippocampus in normal mice. Y-maze test: (A) spontaneous alternation (\%) and (B) number of arm entries. NR1: (C) mRNA expression and (D) protein levels. NR2A: (E) mRNA expression and (F) protein levels. NR2B: (G) mRNA expression and $(\mathrm{H})$ protein levels. GluR1: (I) mRNA expression and $(\mathrm{J})$ protein levels. GluR2: $(\mathrm{K})$ mRNA expression and $(\mathrm{L})$ protein levels. Synaptophysin: (M) mRNA expression and (N) protein levels. PSD-95: (O) mRNA expression and (P) protein levels. Results are expressed as mean \pm SE for $3-7$ mice. ${ }^{*} \mathrm{P}<0.05,{ }^{* *} \mathrm{P}<0.01$ versus vehicle. AMPA, $\alpha$-amino-5-methyl-3-hydroxy-4-isoxazole propionate; CX3CR1, CX3C chemokine receptor 1; mRNA, messenger RNA; NMDA, N-methyl-D-aspartate; PSD-95, postsynaptic density protein 95.

lean rats. ${ }^{910}$ LTP is predominantly regulated by glutamate receptors, such as NMDA and AMPA receptors, and is considered to be a form of synaptic plasticity that underlies learning and memory in the hippocampus. ${ }^{8}$ Activation of CX3CR1 on microglia within the CA1 region of the hippocampus triggers the release of adenosine that in turn, via the activation of type A2 adenosine receptors, increases the release of D-serine as a coagonist for NMDA receptors from the glia, leading to a potentiation of NMDA function, suggesting that fractalkine-CX3CR1 signaling may enhance LTP as well as learning and memory processes. ${ }^{37}$ On the other hand, CX3CR1-deficient mice exhibit impaired LTP in the hippocampus. ${ }^{20}$ These findings suggest that the cognitive impairment observed in DIO mice may be due to decreased fractalkine-CX3CR1 signaling in the hippocampus.

Recent observations demonstrated that circulating and brain IGF-1 modulates brain activities such as neuroprotection, neurogenesis and neuronal excitability. ${ }^{13}$ Serum IGF-1-deficient mice exhibit both cognitive decline and impaired hippocampal LTP. ${ }^{38}$ The present study demonstrated that plasma IGF-1 levels, but not hippocampal IGF-1 levels, were significantly decreased in DIO mice compared with CD mice. Moreover, the present study showed that the administration of the IGF-1 receptor inhibitor PPP significantly decreased mRNA expression of fractalkine and CX3CR1 in the hippocampus, providing different lines of evidence that bloodborne IGF-1 is a potent positive regulator for fractalkineCX3CR1 signaling in the brain.

In the present study, BDNF levels in the hippocampus were significantly decreased in DIO mice compared with $\mathrm{CD}$ mice, consistent with previous reports. ${ }^{347}$ In addition, the administration of the BDNF receptor TrkB antagonist ANA-12 significantly decreased fractalkine and CX3CR1 levels in the hippocampus in normal mice. The mRNA and protein expression of fractalkine and CX3CR1 is significantly decreased in the hippocampus of BDNFdeficit mice. ${ }^{39}$ Together, these findings suggest that HFD induces a significant decrease in hippocampal BDNF 
(A) Fractalkine mRNA

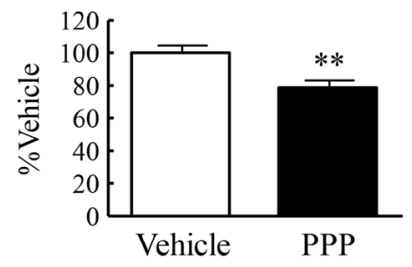

(C) Fractalkine mRNA

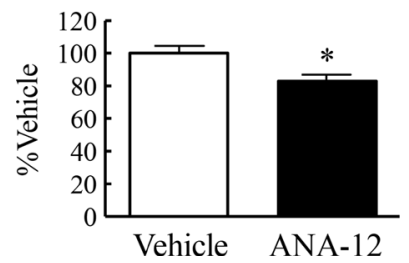

(B) CX3CR1 mRNA

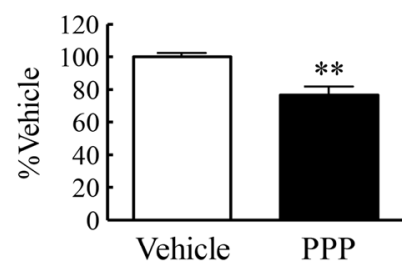

(D) $\mathrm{CX} 3 \mathrm{CR} 1 \mathrm{mRNA}$

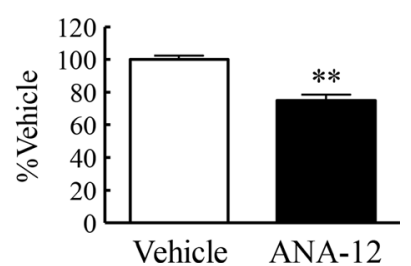

Figure 5 Regulatory effects of IGF-1 and BDNF on mRNA expression of fractalkine and CX3CR1 in the hippocampus of normal mice. PPP: (A) fractalkine mRNA expression and (B) CX3CR1 mRNA expression. ANA-12: (C) fractalkine mRNA expression and (D) CX3CR1 mRNA expression. Results are expressed as mean $\pm S E$ for $12-13$ mice. ${ }^{*} P<0.05$, ${ }^{* *} \mathrm{P}<0.01$ versus vehicle. BDNF, brain-derived neurotrophic factor; CX3CR1, CX3C chemokine receptor 1; IGF-1, insulin-like growth factor-1; mRNA, messenger RNA; PPP, picropodophyllin.

levels, which in turn decreases fractalkine and CX3CR1 levels in the hippocampus.

A recent study demonstrated that the detrimental effects of HFD consumption on learning and memory may be mediated in part by the alterations of glutamate receptors, such as NMDA and AMPA receptors. ${ }^{3440-42}$ HFD feeding decreases hippocampal NR2B of NMDA receptor subunits and GluR-1 of AMPA receptor subunits. ${ }^{434}$ The present study also demonstrated a significant decrease in NR1, NR2A and GluR1 in the hippocampus of DIO mice. Moreover, the present study demonstrated that CX3CR1 antagonist-treated mice also exhibited a significant decrease in NR2A and GluR1 in the hippocampus coincident with significant cognitive deficits, similar to DIO mice. Presynaptic and postsynaptic machinery associated with synapse formation and activity is involved in learning and memory processes. Compared with control mice, DIO mice exhibit abnormal expression of factors involved in the synaptic plasticity, such as synaptosomalassociated protein 25 (SNAP-25) and synaptophysin I in the presynaptic site and PSD-95 in the postsynaptic site. ${ }^{569101443}$ HFD-fed animals exhibit cognitive decline in a water maze test and step-through task, and a decrease in synapsin 1 and PSD-95 in the hippocampus and cerebral cortex. ${ }^{3}{ }^{14}$ Further, HFD-fed mice display impaired hippocampal-dependent memory in the Y-maze test, and reduced SNAP-25, PSD-95 and syntaxin-4, but not synaptophysin, in the hippocampus. ${ }^{6}$ In the present study, DIO mice exhibited a significant decrease in synaptophysin and PSD-95 levels in the hippocampus compared with CD mice. Synaptophysin is presynaptic vesicle marker, and PSD-95 interacts with ion channels, membrane

receptors, cytoskeletal components, and intracellular signaling molecules, ${ }^{4546}$ and increases AMPA receptor currents by selectively delivering GluR1-containing receptors to synapses, thus mimicking LTP. ${ }^{47}$ These findings suggest that the synaptic dysfunction might be an important contributor to the hippocampal-dependent spatial memory impairment in DIO mice. ${ }^{6}$ Furthermore, in the present study, the administration of a CX3CR1 antagonist induced a significant decrease in PSD-95 in the hippocampus of normal mice compared with vehicletreated mice, the same changes observed in the hippocampus of DIO mice. Moreover, the same changes in the NMDA receptor subunits, AMPA receptor subunits, and synaptic markers were observed in both DIO mice and CX3CR1 antagonist-treated mice, indicating that HFD feeding induced impaired fractalkine-CX3CR1 signaling in the hippocampus, leading to synaptic deterioration and resulting in impaired cognition.

There is convincing evidence that enhanced inflammation in both the brain and peripheral organs is strongly involved in several dysfunctions associated with HFD feeding for a long time. ${ }^{48}$ In this regard, fractalkineCX3CR1 signaling is demonstrated to regulate the inflammatory responses of macrophage and microglia. ${ }^{20} 4950$ Especially, enhanced inflammation in both the brain and peripheral organs induced by HFD feeding has been reported to influence cognitive function..$^{20} 314950$ Therefore, enhanced inflammation may be an another pivotal event to regulate processing of learning and memory in obesity.

The present study revealed that long-term HFD feeding in mice may induce impaired fractalkine-CX3CR1 signaling in the corticolimbic system, leading to defective synaptic plasticity and subsequently to cognitive impairment in DIO mice. Taken together, these findings provide compelling evidence that neuron-microglia interactions, especially those underpinned by fractalkineCX3CR1 signaling, play crucial roles in brain dysfunction associated with obesity.

Acknowledgements We wish to thank the Joint Research Laboratory, Kagoshima University Graduate School of Medical and Dental Sciences, for use of their facilities. This work was supported by the Institute of Laboratory Animal Science Research Support Center, Kagoshima University.

Contributors NK, GK, and NY-G performed the experiments, contributed to discussions, and wrote the manuscript. EN performed the experiments. Al and AA contributed to discussions and reviewed and edited the manuscript. NK is the guarantor of this work and as such had full access to all the data in this study and takes responsibility for the integrity of the data and the accuracy of the data analysis.

Funding This study was supported by a Grant-in-Aid for Scientific Research from the Ministry of Education, Culture, Sports, Science and Technology in Japan (16H06404 to Al).

Competing interests None declared.

Patient consent for publication Not required.

Ethics approval All experiments were performed in accordance with the guidelines established by the Institute of Laboratory Animal Science Research Support Center at Kagoshima University and approved by the Kagoshima University Institutional Animal Care and Use Committee (protocol nos. MD18079, MD18080 and MD20007) for the Care and Use of Laboratory Animals. 
Provenance and peer review Not commissioned; externally peer reviewed.

Data availability statement All data relevant to the study are included in the article.

Open access This is an open access article distributed in accordance with the Creative Commons Attribution Non Commercial (CC BY-NC 4.0) license, which permits others to distribute, remix, adapt, build upon this work non-commercially, and license their derivative works on different terms, provided the original work is properly cited, appropriate credit is given, any changes made indicated, and the use is non-commercial. See: http://creativecommons.org/licenses/by-nc/4.0/.

ORCID iD

Namiko Kawamura http://orcid.org/0000-0002-9206-2844

\section{REFERENCES}

1 Elias MF, Elias PK, Sullivan LM, et al. Lower cognitive function in the presence of obesity and hypertension: the Framingham heart study. Int J Obes Relat Metab Disord 2003;27:260-8.

2 Whitmer RA, Gunderson EP, Barrett-Connor E, et al. Obesity in middle age and future risk of dementia: a 27 year longitudinal population based study. BMJ 2005;330:1360-4.

3 Molteni R, Barnard RJ, Ying Z, et al. A high-fat, refined sugar diet reduces hippocampal brain-derived neurotrophic factor, neuronal plasticity, and learning. Neuroscience 2002;112:803-14.

4 Wu A, Ying Z, Gomez-Pinilla F. The interplay between oxidative stress and brain-derived neurotrophic factor modulates the outcome of a saturated fat diet on synaptic plasticity and cognition. Eur $J$ Neurosci 2004;19:1699-707.

5 Arnold SE, Lucki I, Brookshire BR, et al. High fat diet produces brain insulin resistance, synaptodendritic abnormalities and altered behavior in mice. Neurobiol Dis 2014;67:79-87.

6 Lizarbe B, Soares AF, Larsson S, et al. Neurochemical modifications in the hippocampus, cortex and hypothalamus of mice exposed to long-term high-fat diet. Front Neurosci 2018;12:985.

7 Yamada-Goto N, Katsuura G, Ochi Y, et al. Impairment of fearconditioning responses and changes of brain neurotrophic factors in diet-induced obese mice. J Neuroendocrinol 2012;24:1120-5.

8 Neves G, Cooke SF, Bliss TVP. Synaptic plasticity, memory and the hippocampus: a neural network approach to causality. Nat Rev Neurosci 2008;9:65-75.

9 Wang Z, Ge Q, Wu Y, et al. Impairment of long-term memory by a short-term high-fat diet via hippocampal oxidative stress and alterations in synaptic plasticity. Neuroscience 2020;424:24-33.

10 Stranahan AM, Norman ED, Lee K, et al. Diet-Induced insulin resistance impairs hippocampal synaptic plasticity and cognition in middle-aged rats. Hippocampus 2008;18:1085-8.

11 Poo MM. Neurotrophins as synaptic modulators. Nat Rev Neurosci $2001 ; 2: 24-32$

12 Korte M, Carroll P, Wolf E, et al. Hippocampal long-term potentiation is impaired in mice lacking brain-derived neurotrophic factor. Proc Natl Acad Sci U S A 1995;92:8856-60

13 Jones JI, Clemmons DR. Insulin-Like growth factors and their binding proteins: biological actions. Endocr Rev 1995;16:3-34.

14 Liu Y, Fu X, Lan N, et al. Luteolin protects against high fat dietinduced cognitive deficits in obesity mice. Behav Brain Res 2014;267:178-88.

15 Maciejewski-Lenoir D, Chen S, Feng L, et al. Characterization of fractalkine in rat brain cells: migratory and activation signals for CX3CR-1-expressing microglia. J Immunol 1999;163:1628-35.

16 Nishiyori A, Minami M, Ohtani Y, et al. Localization of fractalkine and CX3CR1 mRNAs in rat brain: does fractalkine play a role in signaling from neuron to microglia? FEBS Lett 1998;429:167-72.

17 Imai T, Hieshima K, Haskell C, et al. Identification and molecular characterization of fractalkine receptor CX3CR1, which mediates both leukocyte migration and adhesion. Cell 1997;91:521-30.

18 Réaux-Le Goazigo A, Van Steenwinckel J, Rostène W, et al. Current status of chemokines in the adult CNS. Prog Neurobiol 2013;104:67-92.

19 Sheridan GK, Wdowicz A, Pickering M, et al. Cx3Cl1 is up-regulated in the rat hippocampus during memory-associated synaptic plasticity. Front Cell Neurosci 2014;8:233.

20 Rogers JT, Morganti JM, Bachstetter AD, et al. Cx3Cr1 deficiency leads to impairment of hippocampal cognitive function and synaptic plasticity. J Neurosci 2011;31:16241-50.

21 Nakao K, Katsuura G, Morii N, et al. Inhibitory effect of centrally administered atrial natriuretic polypeptide on the brain dopaminergic system in rats. Eur J Pharmacol 1986;131:171-7.
22 King DL, Arendash GW. Behavioral characterization of the Tg2576 transgenic model of Alzheimer's disease through 19 months. Physiol Behav 2002;75:627-42.

23 Kitamura A, Fujita Y, Oishi N, et al. Selective white matter abnormalities in a novel rat model of vascular dementia. Neurobiol Aging 2012;33:1012.e25-1012.e35.

24 Lalonde R. The neurobiological basis of spontaneous alternation. Neurosci Biobehav Rev 2002;26:91-104.

25 Sarnyai Z, Sibille EL, Pavlides C, et al. Impaired hippocampaldependent learning and functional abnormalities in the hippocampus in mice lacking serotonin(1A) receptors. Proc Natl Acad Sci U S A 2000;97:14731-6.

26 Yamada N, Katsuura G, Tatsuno I, et al. Orexins increase mRNA expressions of neurotrophin-3 in rat primary cortical neuron cultures. Neurosci Lett 2009;450:132-5.

27 Yamada N, Katsuura G, Ochi Y, et al. Impaired CNS leptin action is implicated in depression associated with obesity. Endocrinology 2011;152:2634-43.

28 Karlström S, Nordvall G, Sohn D, et al. Substituted 7-amino-5-thiothiazolo[4,5-d]pyrimidines as potent and selective antagonists of the fractalkine receptor (CX3CR1). J Med Chem 2013;56:3177-90.

29 Menu E, Jernberg-Wiklund H, Stromberg T, et al. Inhibiting the IGF-1 receptor tyrosine kinase with the cyclolignan ppp: an in vitro and in vivo study in the 5T33MM mouse model. Blood 2006;107:655-60.

30 Shirayama Y, Yang C, Zhang J-chun, et al. Alterations in brainderived neurotrophic factor (BDNF) and its precursor proBDNF in the brain regions of a learned helplessness rat model and the antidepressant effects of a TrkB agonist and antagonist. Eur Neuropsychopharmacol 2015;25:2449-58.

31 Cordner ZA, Tamashiro KLK. Effects of high-fat diet exposure on learning \& memory. Physiol Behav 2015;152:363-71.

32 Smith E, Hay P, Campbell L, et al. A review of the association between obesity and cognitive function across the lifespan: implications for novel approaches to prevention and treatment. Obes Rev 2011:12:740-55.

33 Farr SA, Yamada KA, Butterfield DA, et al. Obesity and hypertriglyceridemia produce cognitive impairment. Endocrinology 2008;149:2628-36.

$34 \mathrm{Kim}$ JJ, Koo JW, Lee HJ, et al. Amygdalar inactivation blocks stressinduced impairments in hippocampal long-term potentiation and spatial memory. J Neurosci 2005;25:1532-9.

35 Ikegaya Y, Saito H, Abe K. Dentate gyrus field potentials evoked by stimulation of the basolateral amygdaloid nucleus in anesthetized rats. Brain Res 1996;718:53-60

36 Quirk GJ, Likhtik E, Pelletier JG, et al. Stimulation of medial prefrontal cortex decreases the responsiveness of central amygdala output neurons. J Neurosci 2003;23:8800-7.

37 Scianni M, Antonilli L, Chece G, et al. Fractalkine (CX3CL1) enhances hippocampal N-methyl-D-aspartate receptor (NMDAR) function via D-serine and adenosine receptor type $A 2$ (A2aR) activity. $J$ Neuroinflammation 2013:10:108.

38 Jiang J, Lv X, Wu X, et al. Downregulation of circulating insulin-like growth factor 1 contributes to memory impairment in aged mice after sevoflurane anesthesia. Behav Pharmacol 2017;28:238-43.

39 Wang D-D, Tian T, Dong Q, et al. Transcriptome profiling analysis of the mechanisms underlying the BDNF Val66Met polymorphism induced dysfunctions of the central nervous system. Hippocampus 2014;24:65-78.

40 Kanoski SE, Meisel RL, Mullins AJ, et al. The effects of energyrich diets on discrimination reversal learning and on BDNF in the hippocampus and prefrontal cortex of the rat. Behav Brain Res 2007;182:57-66

41 Xia S-F, Xie Z-X, Qiao Y, et al. Differential effects of quercetin on hippocampus-dependent learning and memory in mice fed with different diets related with oxidative stress. Physiol Behav 2015;138:325-31.

42 Figurov A, Pozzo-Miller LD, Olafsson P, et al. Regulation of synaptic responses to high-frequency stimulation and LTP by neurotrophins in the hippocampus. Nature 1996;381:706-9.

43 Li F, Liu K, Wang A, et al. Cyclic glycine-proline administration normalizes high-fat diet-induced synaptophysin expression in obese rats. Neuropeptides 2019;76:101935.

44 Valladolid-Acebes I, Merino B, Principato A, et al. High-Fat diets induce changes in hippocampal glutamate metabolism and neurotransmission. Am J Physiol Endocrinol Metab 2012;302:E396-402

45 Garner CC, Nash J, Huganir RL. Pdz domains in synapse assembly and signalling. Trends Cell Biol 2000;10:274-80.

46 Sheng M, Sala C. Pdz domains and the organization of supramolecular complexes. Annu Rev Neurosci 2001;24:1-29. 
47 Schnell E, Sizemore M, Karimzadegan S, et al. Direct interactions between PSD-95 and stargazin control synaptic AMPA receptor number. Proc Natl Acad Sci U S A 2002;99:13902-7.

48 Thaler JP, Yi C-X, Schur EA, et al. Obesity is associated with hypothalamic injury in rodents and humans. $J$ Clin Invest 2012;122:153-62.
49 Cope EC, LaMarca EA, Monari PK, et al. Microglia play an active role in obesity-associated cognitive decline. $J$ Neurosci 2018;38:8889-904.

50 Polyák A, Ferenczi S, Dénes A, et al. The fractalkine/Cx3CR1 system is implicated in the development of metabolic visceral adipose tissue inflammation in obesity. Brain Behav Immun 2014;38:25-35. 